\title{
CARACTERIZACIÓN DE LA COMPETENCIA. USO COMPRENSIVO DEL CONOCIMIENTO CIENTÍFICO EN ESTUDIANTES DE BÁSICA SECUNDARIA
}

\section{CHARACTERIZATION OF COMPETENCE COMPREHENSIVE USE OF SCIENTIFIC KNOWLEDGE IN SECONDARY BASIC STUDENTS}

\author{
Jhon Raúl Soto Jiménez ${ }^{1}$ \\ Universidad de Córdoba, Montería, Colombia.

\section{Elvira Patricia Flórez Nisperuza ${ }^{2}$} \\ Universidad de Córdoba, Montería, Colombia. \\ Karen Patricia Agudelo Arteaga ${ }^{3}$ \\ Universidad de Córdoba, Montería, Colombia.
}

\section{RESUMEN}

Este artículo de reflexión presenta los resultados de la fase exploratoria de un proyecto de investigación de maestría en didáctica de las ciencias naturales de la Universidad de Córdoba, denominado “Desarrollo de un entorno blended learning para el fortalecimiento de la competencia uso comprensivo del conocimiento científico", cuyo propósito fue identificar el 
nivel de competencia del uso comprensivo del conocimiento científico en los educandos. En la pesquisa se empleó el enfoque cualitativo, con método descriptivo, con la aplicación de las técnicas: observación a participantes y cuestionario. Los resultados demuestran que se requiere fortalecer el nivel de competencia en las habilidades de identificar, asociar y analizar para la comprensión del conocimiento científico, en ese sentido se propone una estrategia didáctica para fortalecer la competencia.

PALABRAS CLAVE: aprendizaje, enseñanza, resolución de problemas, tic

\section{ABSTRACT}

This reflection article presents the results of the exploratory phase of a research project for a master's degree in didactics of natural sciences at the University of Córdoba, called "Development of a Blended Learning Environment to strengthen the Competence "Comprehensive Use of Scientific Knowledge", the purpose of which was to identify the level of competence in the comprehensive use of scientific knowledge in students. In the research, the qualitative approach was used, with the descriptive method, with the application of the techniques: Observation of participants and questionnaire The results required require the level of competence in the skills of identification, association and analysis for the understanding of scientific knowledge, in this sense a didactic strategy is proposed to strengthen competence.

KEYWORDS: learning, teaching, problem solving, tic.

\section{INTRODUCCIÓN}

En los últimos años se viene presentando una problemática en la Institución Educativa Comfacor "Jaime Exbrayat" de la ciudad de Montería en los grados octavo, la que, se ha evidenciado, a través, de conversaciones y observaciones realizadas a los estudiantes, develando una suma de limitaciones como: la falta de motivación por las ciencias, y correspondiente desinterés por los tópicos de la asignatura, y la falta de apropiación conceptual, por parte de los estudiantes, en temáticas abordadas en los años anteriores. Se considera que estas pueden estar relacionadas con la forma de enseñanza tradicional y las pocas estrategias didácticas por parte de los docentes que dirigen estos cursos. Tal situación, se conecta con las debilidades de los estudiantes en competencias del área de ciencias naturales, específicamente en la competencia uso comprensivo del conocimiento científico, en habilidades como: identificar, asociar, analizar, (Castro y Ramírez, 2013; Coronado y Arteta, 2015; Arrieta, Raillo y Rodríguez, 2017 y Gutiérrez, 2018).

Esto se confirma con los resultados arrojados por las Pruebas Saber en el año 2016 en las cuales los estudiantes de quinto grado presentaron dificultad representativa en la competencia uso comprensivo del conocimiento científico, de igual forma, en el año anterior en la última prueba interna aplicada por Pardo Editores (2019), continúa la debilidad.

A partir de las debilidades y fortalezas encontradas en los educandos, se podría afirmar que la aplicación de nuevas estrategias didácticas en los grados octavos, generaría en los estudiantes la búsqueda de empatía, motivación, curiosidad por las ciencias, aprendizaje significativo, fortalecer habilidades como: identificar, asociar y analizar en la competencia uso del conocimiento científico, y, a su vez, con los nuevos aprendizajes propiciar el desarrollo de otras competencias científicas en las ciencias naturales.

En efecto, dadas las condiciones expuestas en la problemática se han venido dando los primeros pasos para al mejoramiento de las estrategias didácticas, en pro de un mayor entendimiento de la problemática, así como también, buscar 
una posible solución y de la cual surge el interrogante: ¿Cómo desarrollar un entorno blended learning para el fortalecimiento de la competencia uso comprensivo del conocimiento científico en estudiantes de grado de octavo en la Institución educativa Comfacor Jaime Exbrayat de la ciudad de Montería-Córdoba?

En lo que se refiere a la educación básica secundaria específicamente para ciencias naturales se ha detectado que los docentes encuentran dificultades en los estudiantes en sus pre-saberes y en la apropiación de conceptos, pues estos expresan no tener claridad.

Algunas investigaciones como las de Castro y Ramírez (2013); Arrieta, Raillo y Rodríguez (2017) relacionadas con el desarrollo de competencias científicas encontraron necesidades y debilidades similares en estudiantes de básica secundaria, para añadir también la dificultad de aprender a pensar (Papert, 1980; Nickerson, Perkins \& Smith, 1985; Gimeno y Pérez, 1999; Sánchez, 1991, Acedo, 2013) y poner en práctica los conocimientos. Por esta razón, se hace necesario generar un cambio, crear nuevas estrategias didácticas que hagan propicio y agradable el aprendizaje de las ciencias en los estudiantes, tales como la formulación de problemas, resolución de problemas, ABP, entre otras (Gil y De Guzmán,1993; Tesouro,1995; Acedo,2003b; Arteaga,2001).

Para dar respuesta a lo expuesto, se proponen las Tecnologías de la Información y la Comunicación (TIC) en educación, como herramientas que promuevan ambientes dinámicos, interactivos e innovadores para lograr en los estudiantes aprendan a pensar y a resolver problemas cotidianos y científicos. Autores como Jacovkis (2011); Rivero, Gómez y Abrego, (2013); Romero y Quesada (2014); Cabrera, Rojas y Sánchez, (2016); Valeiras, (2006); Vallejo (2010) resaltan el auge que las TIC tienen en la ciencia y la tecnología, además, de la necesidad de los docentes por estar en la vanguardia de las tecnologías para la enseñanza de las ciencias, y la estrecha relación que existe entre métodos usados y los contenidos a enseñar en el trabajo de las ciencias y a su vez su propio dominio.

Sin embargo, hay que seguir trabajando en cerrar esa brecha de las TIC con algunos docentes que aún no logran integrarlas como una herramienta para mejorar sus estrategias didácticas, debido a que algunos en pleno siglo XXI continúan en la enseñanza de las ciencias de forma tradicional, lo que genera en casos desmotivación, apatía de los estudiantes por las ciencias, teniendo en cuenta la diversidad que encontramos día a día en nuestras aulas, en cuanto a los estilos de aprendizaje (Felder, y Silverman, 1988). Lo que conlleva a un mayor reto para estar actualizados en los desafíos y tendencias de la educación (Soto, Senra y Neira, 2009; Linn, 2002; Dunn, R y Dunn, K, 2002).

Teniendo en cuenta lo anterior, uno de los retos de la investigación sería el diseño e implementación de un entorno blended learning con el que se pretende fortalecer la competencia uso del conocimiento científico basado en la resolución de problemas. Este entorno será sincrónico y asincrónico, sin embargo, la retroalimentación será presencial para un mejor acompañamiento del proceso, no como el caso del e-learning solamente virtual y la retroalimentación son condicionamientos o comentarios del docente para mejorar en su proceso. Algunas investigaciones dentro del ámbito de blended learning y uso de Tic, han empleado hacen uso de diferentes herramientas como entorno b-learning desde objetos virtuales de aprendizaje, plataformas en diferentes asignaturas para el fortalecimiento de competencias científicas, pero, sin dejar a parte la importancia el uso de este dentro del proceso de enseñanza de las ciencias, por tanto, mejora las habilidades del estudiante y lo mantiene motivado al verse familiarizado e incluido en 
el entorno blended learning, donde pueden interactuar con sus compañeros y docente en su proceso de enseñanza aprendizaje (Reverte, 2014; Romero y Quesada, 2014; Villasemil, 2016; Buitrago, W y Buitrago, J, 2017; Salgado, 2017).

Otra parte fundamental en la investigación es la estrategia con la que se pretende fortalecer la competencia uso del conocimiento científico, como lo es, la resolución de problemas, de gran importancia en las ciencias naturales, porque a partir de ella se puede lograr el uso de procesos mentales del estudiante, los cuales propician el desarrollo de habilidades de pensamiento que le permitirán, a la hora de enfrentar una situación problema, identificar, asociar y analizar para encontrar una posible solución con argumentos y de manera crítica. Algunas investigaciones como Melo (2015); Arrieta, Raillo y Rodríguez, (2017); Palomino (2017); y Gutiérrez (2018) confirman que esta metodología ha tenido buenos resultados en el fortalecimiento de las competencias científicas en las ciencias naturales, así como también, el reto de los docentes por la preparación de las actividades enfocadas a la solución de problemas (Pisa, Programme for International Student Assessment ,2012).

De este modo, la finalidad de la investigación es analizar de qué forma el entorno blended learning basado en la resolución de problemas contribuye al desarrollo de la competencia uso comprensivo del conocimiento científico en estudiantes básicas secundarias. En torno que emerge como una posible solución a las dificultades que se evidencian en los estudiantes de grado octavo al identificar, asociar y analizar diferentes situaciones contextualizadas en el área de ciencias naturales. De igual forma, se convertiría en una gran fortaleza en la búsqueda, por parte de los estudiantes de grado octavo, a la solución de problemáticas en el contexto cotidiano a partir de sus conocimientos y sus competencias en el área de ciencias naturales.

\section{COMPETENCIAS EN CIENCIAS NATURALES}

Hablar de competencia sería referirnos a la capacidad que tiene las persona para desenvolverse en cualquier contexto a partir de habilidades especificas según sea el campo de acción. Es por ello que se podría referirse a las competencias científicas como "la capacidad de establecer un cierto tipo de relación con las ciencias" (Hernández, 2005, p.1).

En ese orden de ideas de acuerdo al enfoque socioformativo según Tobón, Pimienta y García (2010) se pueden describir las competencias como la formación que debe tener la persona para descifrar, emitir juicios críticos con argumentos sólidos y a su vez solucionar problemas del contexto en donde sea requerido.

Así mismo, el Instituto Colombiano para el Fomento de la Educación Superior define las competencias como "la capacidad de actuar, interactuar e interpretar de cierto modo" (ICFES, 2007, p.14). Entonces se podría afirmar, que el saber desempeñar en cualquier contexto te hace ser una persona con habilidades para ser competente. En realidad, hablar de competencia serviría para reflexionar críticamente, cómo debe ser la formación de los estudiantes, si en verdad deben ser seres competentes, máquinas para competir con los demás en un campo laboral o seres integrales para mejorar la sociedad.

En el área de Ciencias Naturales en Colombia, en la Prueba Saber $11^{\circ}$ el examen de ciencias naturales evalúa la capacidad que tiene el estudiante de comprender y usar nociones, conceptos y teorías de las ciencias naturales en la solución de problemas. En este, se evalúan competencias entre las cuales se destacan el uso comprensivo del conocimiento científico que se refiere a "la capacidad de comprender y usar nociones, conceptos y teorías de las ciencias naturales en la solución de problemas, y de establecer relaciones entre conceptos y conocimientos adquiridos, y fenómenos que se 
observan con frecuencia" (ICFES, 2018, p.27); seguida por la explicación de fenómenos que alude a "la capacidad de construir explicaciones y comprender argumentos y modelos que den razón de fenómenos, y de establecer la validez o coherencia de una afirmación o de un argumento relacionado con un fenómeno o problema científico" (ICFES, 2018, p.27); por último, la indagación que se refiere a "la capacidad para comprender que, a partir de la investigación científica, se construyen explicaciones sobre el mundo natural" (ICFES, 2018, p.p27-28).

\section{RESOLUCIÓN DE PROBLEMAS}

A menudo, pensar es considerado casi como sinónimo de solucionar problemas. Garrett cita a Humphrey (1951) al plantear que pensar es lo que ocurre cuando un organismo encuentra, reconoce y soluciona un problema; no obstante, se podría decir que "solucionar problemas es un elemento necesario dentro de la definición de pensar, entonces estamos considerando que cualquier actividad que falle en encontrar la solución cae fuera del campo del pensamiento" (Garrett, 1988, p.225).

La resolución de problemas implica de una muy buena implementación y orientación por parte del docente con las actividades pertinentes en estudiante, ya que no es sencillo que el estudiante asocie, analice el problema para poder pensar y resolver este, por tal motivo el éxito de la metodología en la resolución de problemas está en las actividades pertinentes y en la dirección efectiva del docente en estas.

Es por ello que plantear "la enseñanza por resolución de problemas da importancia a los procesos de pensamiento del aprendizaje de los estudiantes" (De Guzmán, 2007, p.54). En ese orden de ideas, en un estudio acerca de investigaciones en resolución de problemas se afirma que "el profesor de un aula quien se pregunta qué tipo de problemas incluye en su asignatura para mejorar el interés de sus alumnos" (Gangoso, 2016, p.8).

Entonces hablar de resolución de problemas implica la preparación pertinente de actividades que propicien el desarrollo de solución de problemas, pero también el fortalecimiento de habilidades de pensamiento en el estudiante a partir de las actividades orientadas en la problemática.

Ahora bien, la enseñanza por resolución de problemas es de suma importancia para el estudiante y su proceso en el desarrollo de pensamiento, tal como afirma De Guzmán, (2007) quien sustenta que los procesos de pensamientos del sujeto se caracterizan por aspectos como: la transferencia, la aplicación de los procesos mentales, la autonomía y la conciencia, para lograr transformar, habilidades para identificar un problema, asociar su aprendizaje para intentar resolverlo y analizar de una forma crítica y severa para llegar a una solución pertinente ante la problemática en su actuación en cualquier contexto.

Para finalizar, se debe refirmar la importancia de la resolución de problemas en el proceso de enseñanza de las ciencias: esta estrategia juega un papel fundamental en los procesos mentales de los estudiantes a causa de que mediante el proceso de actividades orientadas al contexto, de lo simple a lo complejo y mediante situaciones problemas cotidianas, aterrizadas a la realidad del educando, genera en primer lugar aflorar debilidades en conceptos o cómo enfrentar un problema, pero de igual forma, le permite en el desarrollo por la búsqueda de la solución del problema, explorar e ir más allá en sus procesos mentales, motivo por el cual las habilidades de pensamiento se verán estimuladas y le permitirán al estudiante buscar alternativas de solución cada vez de manera sencilla de acuerdo en que medida se vea ejercitado con el tipo de actividades pertinentes a la resolución de problemas. 


\section{BLENDED LEARNING}

Los entornos de aprendizaje virtual han sido una buena herramienta en los procesos de enseñanza aprendizaje, sin embargo, muchas veces se queda el proceso solo en actividades virtuales, en las cuales el estudiante es un receptor de información y pocas veces se ve la retroalimentación del proceso por parte del docente, aclarando esto desde el punto del e-learning. En los últimos años donde las TIC han tenido el auge en la educación se toma mano del b-learning un modelo educativo centrado entre la virtualidad del ambiente de aprendizaje y la retroalimentación docente-estudiante en la parte presencial para llevar a cabo un proceso de enseñanza aprendizaje significativo. De igual forma autores como Zepeda, Díaz, Salcedo, y Tapia (2018); Bartolomé, (2004), Bartolomé, (2008) sostienen que el blended learning como un modelo de combinación entre el aprendizaje virtual y la enseñanza presencial, pero añadiendo, los recursos y medios tecnológicos ajustados a un contexto educativo requerido.

Es preciso aclarar que el b-learning se utiliza de diferentes maneras a casusa de que la virtualidad del entorno de aprendizaje puede venir dada por medio de objetos virtuales de aprendizajes, ambientes virtuales de aprendizaje, y a su vez, de manera semejante, plataformas por mencionar algunas como Edmodo, Mangus, Schoology; las cuales te brindan ventajas en la virtualidad, tales como: el seguimiento en tiempo real, programación de actividades, chat entre los estudiantes y el docente, sin embargo, también encontramos limitaciones como la falta de aparatos tecnológicos en las instituciones, hogares, personas las cuales se vería interrumpido el proceso de enseñanza aprendizaje por parte de los estudiantes.

Para otros autores como Samperio, Hernández, Gutiérrez y Pérez, (2015) el "blended learning utiliza las ventajas de la formación presencial y la virtual, integrándolas en un solo modelo educativo, donde el docente combina sus habilidades docentes para implementar las mejores estrategias de enseñanza para lograr el aprendizaje significativo en los alumnos" (p.7).

Teniendo en cuenta las postura de los autores, para el caso de este estudio el uso del entorno b-learning fue muy relevante, en cuanto, que se hizo uso de la plataforma educativa Edmodo, dentro de la cual se diseñó el entorno de aprendizaje a partir de la temática de reproducción en seres vivos, con sus respectivas actividades enfocadas a la resolución de problemas, asimismo, se evidenciaron las ventajas que propicia el entorno $b$, learning como la comunicación asertiva entre el docente y el estudiante mediante el chat, el seguimiento del proceso de enseñanza aprendizaje del estudiante en tiempo real por parte del docente, la interactividad, la cual le genera gran motivación e interés a los educandos y a la hora de la parte presencial se refleja el fortalecimiento de las competencias científicas por parte de los estudiantes, pero también de la estrategia aplicada de las actividades referentes a la temática mediante la resolución de problemas.

Sin embargo, se pueden evidenciar también las limitaciones que genera el entorno $b$, learning por resaltar algunos no hay acceso de internet dentro de la institución, en los hogares de los estudiantes o en los dispositivos móviles, no se cuenta con los aparatos electrónicos para hacer uso del entorno, habilidades para el uso de la herramienta, plataforma caída, perdida de usuarios y contraseñas entre otras.

\section{METODOLOGÍA}

Elestudiosecentróenelenfoquecualitativo,yaque este buscó obtener datos sobre los participantes de la investigación para poder entender e interpretar la realidad del fenómeno a través de la caracterización, diseño y comprensión para generar solución a la problemática planteada en 
este estudio (Hernández, Fernández, y Baptista, 2014). El método que se usó fue el descriptivo (Ñaupas, Palacios, Romero, y Valdivia, 2018), pues se centra en la recopilación de datos a partir de la experiencia y conocimiento de los participantes de la investigación, asimismo, hechos que permitirán conocer la manera como los estudiantes asimilan y aplican sus conocimientos en contextos conocidos y desconocidos. El tipo de caso seleccionado fue intrínseco porque el interés de este estudio se centró en comprender las dificultades que presentaron los estudiantes de grado octavo en la competencia uso compresivo del conocimiento científico; por ende, cada estudiante se convirtió en un caso particular, debido, a que surge la necesidad de conocerlo en profundidad (Stake, 1999; Yin, 2003). A continuación, se denotan las etapas realizadas para el uso de la metodología.

\section{OBSERVACIÓN}

Dado lo anterior, el investigador utilizó inicialmente la técnica de observación, cuyo instrumento fue el protocolo de observación que sirvió para identificar las dificultades en el aprendizaje de los estudiantes de octavo grado en ciencias naturales, y a su vez, sirvió como suministro para la elaboración del cuestionario para identificar el nivel de la competencia uso comprensivo del conocimiento científico de los estudiantes.

\section{PRUEBA PILOTO}

En este aparte se realizó un proceso inicial para definir la muestra, que en este estudio fue no probabilística intencionada, debido a que los casos objetos de estudio presentaron características similares, por ejemplo: nivel bajo en la competencia, cursan el mismo grado, referencia edades, disponibilidad de recursos tecnológicos (Hernández et. al., 2014; Ñaupas, Palacios, Romero, y Valdivia, 2018). La cual está definida por cinco o seis estudiantes de grado octavo de la secundaria de la institución educativa en mención, los cuales hacen referencia a casos particulares objetos de estudio.

Para llevar a cabo la prueba se seleccionó el número piloto, el cual, fue seleccionado a partir de los 21 ítems del cuestionario en construcción para su validación por expertos multiplicado por la muestra intencionada de 5 estudiantes objeto de estudios con resultado de 105 estudiantes aleatoriamente distribuidos en los grados octavos de la institución educativa, con la finalidad de verificación del grado de fiabilidad del instrumento.

\section{VALIDACIÓN POR JUICIO DE EXPERTOS}

Para la validación del cuestionario fue precisado por el uso de juicio de expertos (Herrera y Gallardo s.f.), se le entregó el documento elaborado con el objetivo del instrumento y el respectivo contenido de los ítems dividido en dos sesiones una de preguntas sobre reproducción asexual y otra de reproducción sexual, con un total de 21 ítems de tipo selección múltiple, distribuidos en tres subcategorías 7 ítems de identificar, 7 ítems de asociar y 7 ítems de analizar referentes a la competencia uso comprensivo del conocimiento científico. Cada experto recibió su instrumento de validación con los objetivos, categorías, subcategorías y sus respectivos criterios a evaluar con los que definieron la validez para aplicación o no del instrumento.

\section{IMPLEMENTACIÓN}

Luego de someterse al juicio de expertos el instrumento fue validado con las respectivas observaciones, lo que llevaron como resultado final del instrumento dividido en dos sesiones una de preguntas sobre reproducción asexual y otra de reproducción sexual, con un total de 15 ítems de tipo selección múltiple, distribuidos en tres subcategorías 5 ítems de identificar, 5 ítems de asociar y 5 ítems de analizar. Seguido los ajustes y después de ser validado por un grupo de expertos y se hace uso de la técnica 
del cuestionario con la aplicación a la muestra objeto de estudio (Herrera y Gallardo s.f.), con la finalidad de identificar el nivel de los estudiantes del grado octavo en la competencia uso compresivo del conocimiento científico.

Los datos recopilados fueron el resultado de la identificación del nivel bajo de las subcategorías (identificar, asociar, analizar) de la competencia uso comprensivo del conocimiento científico, además, permitieron dar a conocer las falencias, desmotivación y desinterés que tenían los estudiantes en el área de ciencias naturales. Posterior a la recolección y organización de los datos se un análisis de corte descriptivo.

\section{RESULTADOS}

Con la intención de reflexionar a partir de los resultados que se obtuvieron en el trabajo de campo de la primera fase de estudio, los cuales se relacionan con el nivel de la competencia del uso comprensivo del conocimiento científico que poseen los estudiantes del grado octavo sobre reproducción en seres vivos en la Institución educativa Comfacor Jaime Exbrayat, se presenta este apartado del proceso que se realizó a partir del análisis descriptivo apoyado del cuestionario validado por expertos, basado en 15 ítems sobre reproducción en seres vivos en la competencia Uso comprensivo del conocimiento científico, además de las subcategorías específicas descritas en 5 ítems de identificar, 5 ítems de asociar y 5 ítems de analizar respectivamente.

En la investigación se identificó el bajo nivel de la competencia uso comprensivo del conocimiento científico que poseen los estudiantes del grado octavo sobre reproducción en seres vivos en la Institución educativa Comfacor Jaime Exbrayat, mediante el análisis cualitativo de corte descriptivo de contenido artesanal apoyado en el software Excel.

En adelante se presenta el análisis de cada uno de los casos que son objetos de estudio y que permitieron llegar a esta generalización de los resultados y las posibles acciones de mejoras para el fortalecimiento de la competencia-

Tabla 1. Preguntas de diagnóstico caso 1 subcategorias

\section{ITEMS O PREGUNTAS DE DIAGNOSTICO SOBRE REPRODUCCIÓN EST 1}

\section{SUBCATEGORIAS}

$\begin{array}{llllllllllllllll}\mathrm{N}^{0} & 1 & 7 & 11 & 14 & 15 & 2 & 4 & 8 & 10 & 13 & 3 & 5 & 6 & 9 & 12\end{array}$

\section{ESTUDIANTES}

\begin{tabular}{llllllllllllllll}
\hline 1 & 0 & 0 & 0 & 1 & 1 & 0 & 0 & 0 & 0 & 0 & 0 & 0 & 1 & 0 & 0 \\
\hline 1 & IDENTIFICAR: & 2,0 & ASOCIAR: 0,0 & & ANALIZAR: 1,0 \\
& BAJO & & & & BAJO & & & BAJO & &
\end{tabular}

Fuente: Propia

Del caso 1 se puede inferir que posee un nivel bajo de la competencia "Uso comprensivo del conocimiento científico" en general sobre reproducción en seres vivos, motivo por el cual, el estudiante logra un nivel muy mínimo en reconocer y diferenciar representaciones referentes a la reproducción en seres vivos, asimismo, el estudiante no logra asociar, es decir, no establece relaciones, entre, la información que se le brinda y los datos para resolver un interrogante ante una situación problema referente a los ítems en asociar sobre 
la reproducción en seres vivos, por consiguiente, este caso es relevante, porque, al no poseer la capacidad para identificar y asociar, se le va a presentar la dificultad para analizar cualquier situación propuesta a partir de los ítems específicos de analizar en el diagnóstico, en ese orden de ideas, cabe resaltar el resultado del trabajo de Naranjo, Leiton, y Zúñiga, (2014) del sistema educativo tradicional hacia la formación por competencias en esta investigación se evidencia de manera el panorama de los sistemas educativos de dos países de Latinoamérica, respecto a las competencias científicas y a su vez evalúa el nivel de las competencias científicas en estudiantes.

Asimismo, haciendo un comparativo con nuestro caso 1 los resultados contrastados con los de Costa rica, se evidencia el bajo nivel de las competencias científicas, donde los estudiantes presentan debilidades a la hora de identificar, explicar y utilizar información para llegar a la finalidad de las respuestas para una conclusión precisa.

Tabla 2. Preguntas de diagnóstico caso 2 subcategorias

\section{ITEMS O PREGUNTAS DE DIAGNOSTICO SOBRE REPRODUCCIÓN EST.2}

\section{SUBCATEGORIAS}

\begin{tabular}{llllllllllllllll}
\hline $\mathrm{N}^{\circ}$ & 1 & 7 & 11 & 14 & 15 & 2 & 4 & 8 & 10 & 13 & 3 & 5 & 6 & 9 & 12
\end{tabular}

\section{ESTUDIANTES}

\begin{tabular}{llllllllllllllll}
\hline 2 & 1 & 1 & 1 & 1 & 0 & 1 & 0 & 1 & 0 & 1 & 0 & 0 & 1 & 0 & 0 \\
\hline 2 & IDENTIFICAR: & 4,0 & ASOCIAR: 3,0 & & ANALIZAR: 1,0 \\
& ALTO & & & BAJO & & & BAJO & & &
\end{tabular}

Fuente: Propia

En este caso 2, se puede denotar que el estudiante presenta un nivel bajo a nivel de la competencia "Uso comprensivo del conocimiento científico", en general sobre reproducción en seres vivos, sin embargo, cuenta con fortalezas en reconocer y diferenciar fenómenos, representaciones pertinentes a la reproducción, en la subcategoría de identificar con un nivel alto, denotado en la escala de subcategorías, sin embargo, presenta falencias en establecer relaciones a partir de datos e información, además, que no logra interpretar información relevante a partir de buscar, seleccionar y organizar para llegar a una conclusión correcta. En ese orden de ideas se puede presentar una similitud con el estudio de Melo (2015) en donde el reflejo de los resultados de su trabajo en la búsqueda de la identificación del nivel de competencia a partir de la resolución de problemas se denota que los estudiantes no tienen la habilidad de asociar, por tal motivo lo anterior se puede relacionar con que el estudiante no cuenta con la capacidad de establecer relaciones entre la información y los datos recopilados, asimismo, en la subcategoría analizar donde el estudiante debería lograr tener la capacidad de buscar, recoger, seleccionar, organizar e interpretar información (ICFES, 2018). 
Tabla 3. Preguntas de diagnóstico caso 3 subcategorias

ITEMS O PREGUNTAS DE DIAGNOSTICO SOBRE REPRODUCCIÓN EST.3 SUBCATEGORIAS

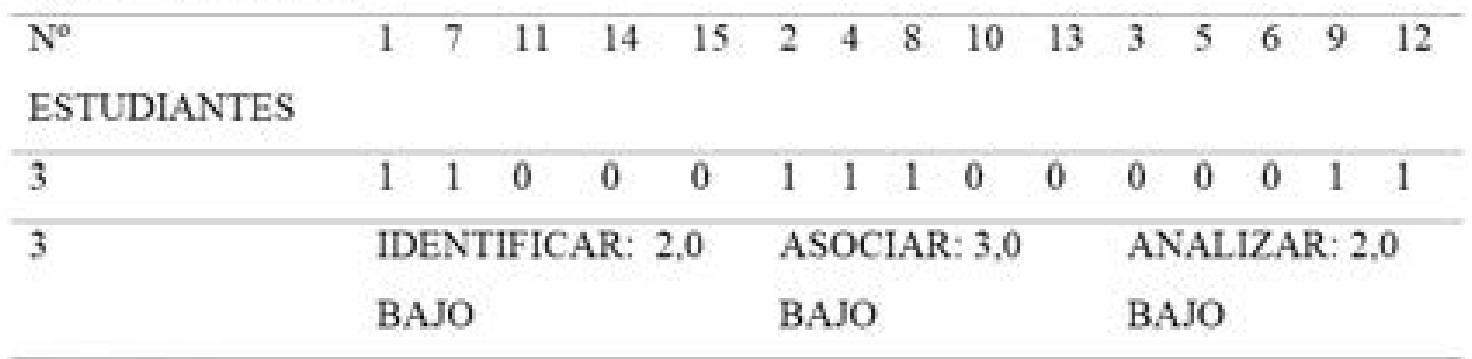

Fuente: Propia.

El caso 3, se identifica el bajo nivel de competencia en el "Uso comprensivo del conocimiento científico", por consiguiente, el estudiante se le dificulta reconocer, diferenciar, establecer relaciones, y, a su vez, interpretar para concluir y llegar a las respectivas soluciones de problemas o situaciones propuestas referentes a la reproducción en seres vivos. De igual forma en los resultados de Naranjo, Leiton, y Zúñiga (2014) en el caso de las competencias donde el estudiante debe estar "familiarizado con procesos de investigación científica, como la experimentación, la observación y análisis de datos, habilidades para argumentar, analizar y sacar conclusiones así como habilidades para reconocer, recordar, e identificar algunos conceptos" (p.152) se puede evidenciar una similitud con los resultados del caso 2 que genera una conclusión que en algunos países de Latinoamérica se vienen presentando falencias en estas habilidades por parte de los estudiantes y en el manejo de estrategias didácticas por parte de los docentes en el quehacer.

Tabla 4. Preguntas de diagnóstico caso 4 subcategorias

\section{ITEMS O PREGUNTAS DE DIAGNOSTICO SOBRE REPRODUCCIÓN EST.4}

\section{SUBCATEGORIAS}

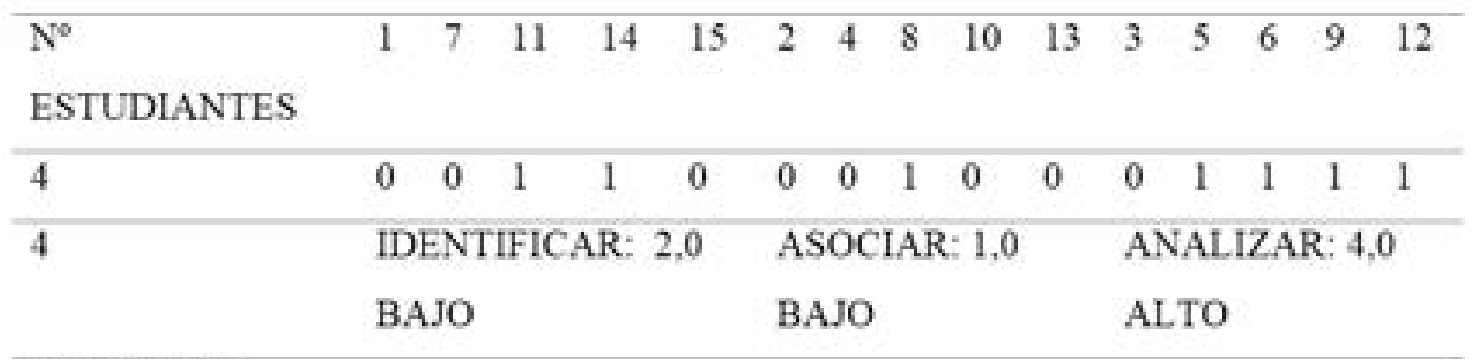

Fuente: Propia. 
El caso 4, es particular, debido que, a nivel de la competencia el estudiante presenta un nivel de desempeño bajo, sin embargo, contempla un nivel alto analizando a partir de situaciones problemas, pero la particularidad es como logra analizar sin poder identificar y asociar, lo que se quiere denotar es que al tener la capacidad de buscar, seleccionar, organizar para llegar a una respuesta conclusa y bien interpretada, se podría inferir que tendría la capacidad para identificar, asociar para poder llegar al análisis pertinente. Algunos estudios como el de Melo (2015) sostienen que los estudiantes en el proceso de analizar presentan dificultad a la hora de enfrentar soluciones problémicas debido a la poca asociación del fenómeno en estudio y el cual deben tener una gama de conceptos donde logren identificar y asociar estos para llegar a una conclusión, sin embrago, para el caso 4 fue fácil como lo evidencian los resultados en el diagnóstico, porque, logró analizar el fenómeno expuesto sobre reproducción en seres vivos infiriendo que hizo la pertinente identificación y asociación de conceptos propios de la naturaleza del fenómeno, sin embargo, es objeto de estudio propiciar a partir de cual estrategia el estudiante llegó a la finalidad de los interrogantes de una forma correcta.

Tabla 5. Preguntas de diagnóstico caso 5 subcategorias

\section{ITEMS O PREGUNTAS DE DIAGNOSTICO SOBRE REPRODUCCIÓN EST.5} SUBCATEGORIAS

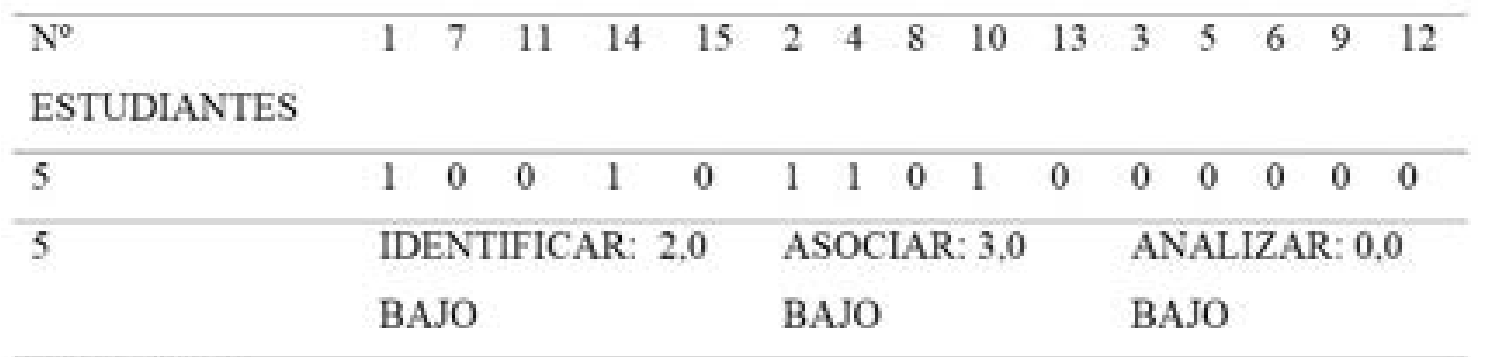

Fuente: Propia.

El caso 5 se identifica el bajo nivel de competencia en el "Uso comprensivo del conocimiento científico" como consecuencia el estudiante no logra relacionar, diferenciar y establecer relaciones referentes a la reproducción en seres vivos, además, de presentar la dificultad para interpretar, a partir de, información o datos presentados para fundamentar una respuesta pertinente al análisis de una situación o cuestionamiento. Un estudio realizado por Salgado (2017), en la ciudad de Montería denota el bajo desempeño de estudiantes en la competencia "Uso comprensivo del conocimiento científico" al momento de aplicar un test diagnostico en ciencias naturales de la misma competencia, lo que permite reforzar el caso 5 donde los estudiantes están presentando dificultad en las habilidades de la competencia a la hora de identificar, asociar y analizar a partir de un fenómeno o temática de estudio.

\section{CONCLUSIONES}

Para resaltar los estudiantes con bajo nivel de la competencia Uso Comprensivo del Conocimiento Científico no alcanzan a reconocer, diferenciar y relacionar conceptos propios y asociados referentes a la reproducción en seres vivos para lograr interpretar la información presentada, para así, fundamentar su respuesta en el análisis de una situación o pregunta dada. De manera similar autores como Salgado, 2017; Arrieta, Raillo y Rodríguez (2017) Naranjo, Leiton, y Zúñiga, 2014 y Castro y Ramírez (2013), sostienen 
que han encontrado dificultades similares en cuanto a las competencias científicas y habilidades como identificar, asociar y analizar en los estudiantes.

Por otra parte, se puede mencionar los hallazgos del caso 4 del cual, presentó bajo nivel en las subcategorías de identificar y asociar, de la competencia en mención, lo que denota, que el estudiante logra reconocer y diferenciar representaciones propias de la naturaleza del fenómeno en estudio, y a su vez, establecer relaciones entre la información y los datos recopilados para encontrar una posible respuesta al interrogante o situación expuesta, sin embargo, mostró un nivel alto en la subcategoría analizar de la competencia uso comprensivo del conocimiento, donde, el estudiante tiene la capacidad de buscar, recoger, seleccionar, organizar e interpretar información (ICFES, 2018).

Si bien es cierto que el estudiante tiene la capacidad de buscar, seleccionar, organizar para llegar a una respuesta conclusa, a través, de una buena interpretación del cuestionamiento, ¿cómo logra analizar sin poder identificar y asociar?

Para ilustrar el caso 4 se podría, corroborar con la investigación de Melo (2015) que sostienen que los estudiantes en el proceso de analizar presentan dificultad a la hora de enfrentar soluciones problemas o cuestionamientos, debido, a la poca asociación del fenómeno en estudio, y el cual deben tener claridad en la conceptualización para lograr identificar y asociar estos, para llegar a una conclusión, situación similar de lo que ocurre en el caso 2, a causa, del nivel alto presentado en la subcategoría identificar pero nivel bajo en las subcategorías de asociar y analizar, por tal motivo, lo anterior se puede relacionar con que el estudiante no posee la capacidad de establecer relaciones entre la información y los datos recopilados (ICFES, 2018), para alcanzar lograr tener la capacidad de analizar para alcanzar una respuesta propicia a partir de la búsqueda, organización y selección de la información.

No obstante, es necesario recalcar que los estudiantes a pesar de estar enfocados en las habilidades de identificar, asociar y analizar de la competencia Uso comprensivo del conocimiento científico, son conscientes de las debilidades en ella, a causa de la falta de actividades enfocadas a la solución de problemas para el fortalecimiento de la competencia.

Finalmente, estos hallazgos sirvieron de base para el diseño de un entorno blended learning basado en la resolución del problema resultado de otro objetivo de esta investigación.

En cuanto al diseño de la herramienta tecnológica para un entorno b- learning sobre reproducción en seres vivos, se seleccionó, la plataforma Edmodo, dado que, es una plataforma tecnológica, educativa, gratuita, $y$, fue creada, para uso en cualquier contexto educativo, además, brinda un espacio propicio para la empatía con el estudiante, debido que, se ven familiarizados con la similitud con algunas redes sociales que permiten muros de publicación, comentarios, likes o me gusta en la plataforma. Esta, también, facilita a los docentes la comunicación sincrónica, con los estudiantes, en un entorno privado y de otro modo, mediante el chat o comentarios en las publicaciones a nivel general y asertivo con toda la clase en el aula virtual (Edmodo, 2020).

Otra característica favorable de la plataforma, es la organización que le proporciona al docente, en el seguimiento del proceso de enseñanza aprendizaje del estudiante en tiempo real, asimismo, la interactividad, que le genera gran motivación e interés a los educandos al momentos de disfrutar todas sus bondades de la plataforma, por tal motivo, a la hora de la parte presencial y retroalimentación, se verá reflejado 
en el fortalecimiento de las competencias científicas por parte de los estudiantes, a partir de la estrategia didáctica aplicada y de las actividades planificadas, referentes a la temática mediante la resolución de problemas (De guzmán, 2007;Gil y De guzmán,1993; Garrett,1988), que en realidad promueven el aprendizaje de los educandos. Ahora bien, la enseñanza por resolución de problemas es de suma importancia para el estudiante y su proceso en el desarrollo de pensamiento, tal como, afirma De Guzmán, (2007) donde los proceso de pensamientos del sujeto y algunas características como: hacer la transferencia, la aplicación de las actividades a procesos mentales, capacidad de ser autónomo, consciente para lograr transformar a partir de su propio pensamiento, y algo muy valioso, ser competente para las ciencias, así como, para la vida diaria en cualquier contexto.

\section{REFERENCIAS BIBLIOGRÁFICAS}

Acedo, M. (2003). Estrategias Cognitivas en la enseñanza del inglés técnico científico: una experiencia. Anales de la Universidad Metropolitana, 3 (2) Recuperado de:

http://prof.usb.ve/macedo/ indexMLAB/ servidor/itc/Documentos/estitc.htm.

Arteaga, E. (2001). El sistema de tareas para el trabajo independiente creativo en la enseñanza de la Matemática en el preuniversitario (Tesis Doctoral). Universidad de Cienfuegos. Cienfuegos, Cuba.

Arrieta, L., Raillo, M., y Rodríguez, A. (2017). Estrategias didácticas para el desarrollo de competencias científicas en el grado octavo de la Institución Educativa Inem Lorenzo María Lleras de Montería (Tesis de Maestría). Universidad de Santo Tomás, Montería, Colombia. Recuperado de http://repository.usta. edu.co/handle/11634/10098

Bartolomé, A. (2008). Entornos de aprendizaje mixto en Educación Superior. Revista Iberoamericana de Educación a Distancia, 11(1), 15-51. Recuperado de http://revistas.uned.es/index.php/ried/ article/view/955

Bartolomé, A. (2004). Blended Learning. Conceptos Básicos. Pixel-Bit, 23, 7-20. https://idus.us.es/xmlui/ bitstream/handle/11441/55455/ B le nded \% 20 le arning. $\% 20$ Conceptos $\% 20$ b\% c $3 \%$ a 1 sicos. pdf? sequence $=1 \&$ isAllowed $=y$

Buitrago, W., y Buitrago, J. (2017). Desarrollo de las habilidades de probabilidad e incertidumbre mediante un ambiente B-Learning en estudiantes de 10 a 12 años de dos colegios públicos de Bogotá (Tesis de Maestría). Universidad de La Sabana, Bogotá, Colombia. Recuperado de:

https://intellectum.unisabana.edu.co/ handle/10818/29706

Cabrera, J., Rojas, F., y Sánchez, I. (2016). Uso De Objetos Virtuales De Aprendizaje Ovas Como Estrategia De Enseñanza Aprendizaje Inclusivo Y Complementario A Los Cursos Teóricos - Prácticos. Revista Educación en Ingeniería, 11(22), 4-12. Recuperado de: https://doi. org/10.26507/rei.v11n22.602

Castro, A., y Ramírez, R. (2013). Enseñanza De Las Ciencias Naturales Para El Desarrollo De Competencias Científicas. Amazonia Investiga, 2(3). Recuperado de http://www.udla.edu.co/revistas/index. php/amazonia-investiga/article/view/31 
Coronado, M, y Arteta, J. (2015). Competencias científicas que propician docentes de Ciencias naturales. Zona Próxima, (23), 131-144.

Recuperado de: https://www.redalyc.org/ articulo.oa? id=853/85344718009

De Guzmán, M. (2007). Enseñanza de las Ciencias y la Matemática. Revista Iberoamericana de Educación 43, 19-58. Recuperado de: https://rieoei.org/RIE/article/view/750

Dunn, R. y Dunn, K. (2002) “La comprensión del estilo de aprendizaje y su necesidad en el diagnóstico y prescripción individualizados". En Enseñanza y estilo individual de aprendizaje, Madrid, España.

Edmodo. (2020). Conoce sobre Edmodo.

Recuperado de: https://go.edmodo. com/sobre-edmodo/?lang=es

Felder, R. M., \& Silverman, L. K. (1988). Estilos de aprendizaje y de enseñanza en la educación de ingeniería. Ing. Educación, 78(7), 674-681.

Gangoso, Z. (2016). Investigaciones En Resolución De Problemas En Ciencias. Investigações em Ensino de Ciências, 4(1), 7-50. Recuperado de:

https://www.if.ufrgs.br/cref/ojs/index. php/ienci/article/view/609

Garrett, R. M. (1988). Resolución de problemas y creatividad: Implicaciones para el currículo de ciencias. Enseñanza de las ciencias: revista de investigación y experiencias didácticas, 6(3), 224-230. Recuperado de:

https://www.raco.cat/index.php/Ensenanza/ article/view/51098
Gil, D., y De Guzmán, M. (1993). La enseñanza de las Ciencias y la Matemática. Tendencias e innovaciones. Organización de Estados Iberoamericanos. Madrid, España: Editorial Popular. Recuperado de http:// www.oei.org.co/oeivirt/ciencias.pdf

Gimeno, J. y Pérez, A. (1999). Comprender y transformar la enseñanza. Madrid, España: Morata.

Gutiérrez, C. (2018). Fortalecimiento de las competencias de interpretación y solución de problemas mediante un entorno virtual de aprendizaje. Revista De Investigación, Desarrollo e Innovación, 8(2). Recuperado de:

https://doi.org/10.19053/20278306. v8.n2.2018.7170

Hernández, R, Fernández, C, y Baptista, P. (2014). Metodología De La Investigación (sexta edición). Recuperado de:

http://library1.org/_ads/

E730F5A55CF4BD418343B9263AE1936E

Hernández, C. (2005). Que son las competencias científicas. Recuperado de: http://www. acofacien.org/images/files/encuentros/ directores_de_carrera/i_reunion_de_ directores_de_carrera/ba37e1_que\%20 son $\% 20$ las $\% 20$ competencias $\% 20$ cientificas\%20-\%20c.a.\%20hernandez. pdf

Herrera, L., y Gallardo, M. (s.f). Métodos y técnicas cuantitativas de análisis en la investigación educativa. pp. 72-76 
Instituto Colombiano para la Evaluación de la Educación. (2018). Guías de orientación. Icfes Instituto Colombiano para la Evaluación de la Educación. Recuperado de: http://www2.icfesinteractivo.gov. co/investigacionFormulario/item/2490guias-de-orientacion

Instituto Colombiano para el Fomento de la Educación Superior. (2007). Fundamentación conceptual área de ciencias naturales.

Recuperado de: http://paidagogos.co/pdf/ fundamentacion_ciencias.pdf

Jacovkis, P. (2011). Las TIC en América Latina: Historia e impacto social. Iberoamericana de Ciencia, Tecnología y Sociedad - CTS, 6(18). Recuperado de http://www.redalyc.org/articulo. oa? id=92422639003

Linn, M. (2002). Promover La Educación Científica A Través De Las Tecnologías De La información y comunicación. Enseñanza de las Ciencias, 20 (3), pp. 347-355.

Melo Manrique, L. (2015). El aprendizaje por resolución de problemas una estrategia para el desarrollo de la competencia uso comprensivo del conocimiento científico en estudiantes de grado octavo del colegio El Porvenir. Sede B. Jornada tarde, (Tesis de Maestría). Universidad de La Sabana, Bogotá, Colombia. Recuperado de: https://intellectum. unisabana.edu.co/handle/10818/19784

Naranjo, J., Leiton, R., y Zúñiga, A. (2014). Del sistema educativo tradicional hacia la formación por competencias: Una mirada a los procesos de enseñanza aprendizaje de las ciencias en la educación secundaria de Mendoza Argentina y San José de Costa Rica. Eureka sobre Enseñanza y Divulgación de las Ciencias, 11(2) ,145-159.

Recuperado de:

https://revistas.uca.es/index.php/eureka/ article/view/2872

Nickerson, R. S., Perkins, D. N. \& Smith, E. E. (1985). The teaching of the thinking. Nueva Jersey: Lawrence Erlbaum Associates. Traducción al castellano: Enseñar a pensar. Aspectos de la aptitud intelectual. Barcelona: Paidós, 1987.

Ñaupas, H, Palacios, J, Romero, H, y Valdivia, M. (2018). Metodología de la investigación. Bogotá, Colombia: Ediciones de la U.

Palomino, E. (2017). La aplicación de las fases de resolución de problemas de George Polya en el marco de las rutas de aprendizaje en los estudiantes del III ciclo de la I.E. No 131, (Tesis de especialización). Pontificia Universidad Católica del Perú, San Miguel, Perú. Recuperado de:

http://tesis.pucp.edu.pe/repositorio// handle/123456789/8480

Papert, S. (1980). Mindstorms. Nueva York United of States: Basic Books.

Pardo Editores (2019). Simulacro No 1-4 Aprender para Saber. Recuperado de http://www.helmerpardo.com

Pisa. Programme for International Student Assessment (2012). Resolución de problemas de la vida real Resultados de Matemáticas y Lectura por ordenador. Gobierno de España. pp 114. 
Reverte, J. (2014). Diseño, implementación y validación de un ambiente enriquecido con TIC para el aprendizaje del álgebra en 30 de ESO, (Ph.D. Thesis), Universidad de las Islas Baleares, Palma de Mallorca, España. Recuperado de:

http://www.tdx.cat/handle/10803/28319

Rivero, I., Gómez, M., y Abrego, R. (2013). Tecnologías educativas y estrategias didácticas: Criterios de selección. Revista educación y tecnología, (3), 190206. Recuperado de:

http://revistas.umce.cl/index.php/edytec/ article/view/134

Romero, M., y Quesada, A. (2014). Nuevas tecnologías y aprendizaje significativo de las ciencias. Enseñanza de las ciencias, 32(1), 0101-0115. https://doi. org/10.5565/rev/ensciencias. 433

Salgado, J. (2017). Influencia De Los Proyectos Formativos En Ambientes B-Learning Para El Desarrollo De Competencias Específicas En El Área De Ciencias Naturales, En Estudiantes De Grado Séptimo En La Institución Educativa Cristóbal Colón Montería, (Tesis de Maestría). Universidad de Córdoba, Montería, Colombia.

Samperio, T, Hernández, Gutiérrez, M, y Pérez, D. (2015). Modelo B-learning, como una alternativa innovadora y de éxito en los sistemas educativos de nivel superior, (Tesis de Maestría). Universidad Autónoma del Estado de Hidalgo México, Hidalgo, México. Recuperado de: https://reposital.cuaed.unam. $\mathrm{mx}: 8443 / x m l u i /$ handle/123456789/3862

Sánchez, M. (1991). Desarrollo de habilidades del pensamiento. Procesos básicos del pensamiento. Monterrey, México: Trillas.
Soto, C., Senra, A., y Neira, M. (2009). Ventajas del uso de las TICs en el proceso de enseñanza-aprendizaje desde la óptica de los docentes universitarios españoles. Electrónica de Tecnología Educativa, 29. 1-12. doi: https:// doi.org/10.21556/edutec.2009.29.451

Stake, R. (1999). Investigación con estudio de casos (2da ed.). Morata. Recuperado de http://site.ebrary.com/lib/ interpuertoricosp/Doc?id=10831642

Tesouro, M. (1995). Optimització del rendiment intel.lectual mitjançant instrucció informatitzada. (Tesis doctoral). Universidad Autónoma de Barcelona, Barcelona, España.

Tobón, S., Pimienta, J. y García, J. (2010). Secuencias didácticas: Aprendizaje y Evaluación de Competencias. México: Edit. Pearson.

Valeiras, E. (2006). Las tecnologías de la información y la comunicación integradas en un modelo constructivista para la enseñanza de las ciencias, (Tesis Doctoral). Universidad de Burgos, Burgos, España. Recuperado de http:// hdl.handle.net/10259/70

Vallejo, A., y Montes, A. (2010). Integración de las TIC en la asignatura de Tecnología de Educación Secundaria. Píxel-Bit. Revista de medios y educación, (37), 225-337

Villasevil, F. (2016). Influencia de los multimedia (TIC-TAC) en el proceso enseñanza/ aprendizaje (Ph.D. Thesis,). Universidad Politécnica de Cataluña, Cataluña, España. Recuperado de http://www.tdx. cat/handle/10803/385747 
Yin, R. (2003). Investigación Sobre de Diseño Y Métodos (Vol. 5). United of States: Sage. Recuperado de: https://www.polipub. org/articulo/index/id/24/page/3

Zepeda, G., Diaz, L, Salcedo, M, y Tapia, S. (2018). Una aproximación teórica sobre MOOC, Aula Invertida, y B-Learning: Similitudes y diferencias. Educateconciencia, 20(21). Recuperado de: http://tecnocientifica. com.mx/educateconciencia/index.php/ revistaeducate/article/view/563. 